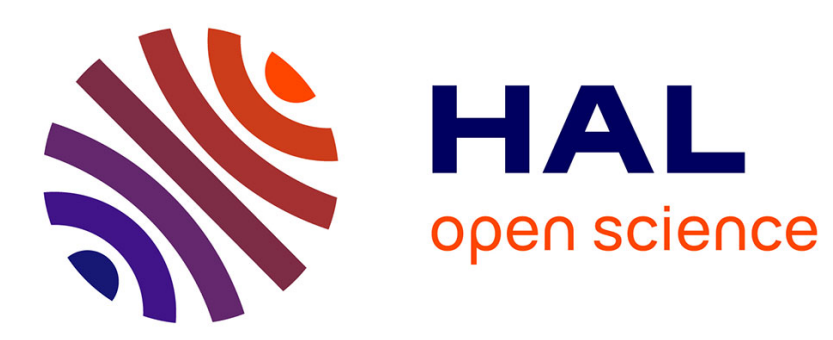

\title{
Structural Mechanism of Close-Packed Polytypes Formation at Displacive Type Phase Transformation in Alloys
}

A. Ustinov

\section{> To cite this version:}

A. Ustinov. Structural Mechanism of Close-Packed Polytypes Formation at Displacive Type Phase Transformation in Alloys. Journal de Physique IV Proceedings, 1996, 06 (C1), pp.C1-75-C1-84. 10.1051/jp4:1996108 . jpa-00254139

HAL Id: jpa-00254139

https://hal.science/jpa-00254139

Submitted on 1 Jan 1996

HAL is a multi-disciplinary open access archive for the deposit and dissemination of scientific research documents, whether they are published or not. The documents may come from teaching and research institutions in France or abroad, or from public or private research centers.
L'archive ouverte pluridisciplinaire HAL, est destinée au dépôt et à la diffusion de documents scientifiques de niveau recherche, publiés ou non, émanant des établissements d'enseignement et de recherche français ou étrangers, des laboratoires publics ou privés. 


\title{
Structural Mechanism of Close-Packed Polytypes Formation at Displacive Type Phase Transformation in Alloys
}

\author{
A.I. Ustinov \\ Institute for Metal Physics, 36 Vernadsky str., Kiev-142, 252680 Ukraine
}

\begin{abstract}
Statistics of one-dimensional disordering of polytypes formed under phase transformation of a displacive type of Co-alloys was studied. Discovered polytypes with a short-range order in close-packed atomic layers allowed to propose a mechanism of their formation by noncoherent displacements in the initial polymorphous modification along close-packed planes. Significant dependence of polytype structures not only on alloying of an alloy but on the kinetics of a polymorphous transformation is considered as an indirect interaction of atomic layers via the impurities subsystem.
\end{abstract}

\section{INTRODUCTION}

Numerous close-packed structures are formed in some metal systems under polymorphous transformations caused by thermal mechanic effect [1-2]. Analyzing these structures we came to the conclusion, that they are polytypes, i.e. they can be described as differently arranged relatively each other actually identical close-packed atomic layers. As far as thermalmechanical behavior of a material (crystallographic reversible elastic or pseudoelastic deformation) is significantly determined by a crystal structure of the initial and final state of a crystal and a type of a diagram strain (temperature)-deformation is determined by a metastable (intermediate) states structure, the reasons and mechanisms of these or that polytypes formation are very important to be found out [3-4].

Similar multitude of structural states were observed for the first time under crystallization $(\mathrm{SiC})$ and ordering $(\mathrm{CuAu})$. Polytype models for these substances were attempted to be applied for explanations of polytype formations and phase transformations of a displacive type. Unfortunately, the explanation of these polytype structure peculiarities (their long-range correlation in atomic layers arrangement, heterostructural state and onedimensionally disorder (ODD), in particular) is appeared to be impossible within these models. Analysis of these approaches demonstrated, that they did not take into account the polytype formation under phase transformation in a solid solution according to a displacement mechanism.

This work analyses the experimental results of polytype structures formation in Coalloys. Polytype structures, their formation and stability prove the necessity of consideration of polymorphous transformation kinetics under sufficient diffuse mobility of atoms of an alloying element (impurity).

\section{CRYSTALLOGRAPHY OF CLOSE-PACKED POLYTYPE FORMATION}

The crystallographic analysis of the initial and final structural states of the simplest polymorphous transformation $3 \mathrm{C}-2 \mathrm{H}$ shows (fig.1), that displacement of each second close- 
packed plane to a vector $\frac{1}{3}\left(\vec{a}_{2}-\vec{a}_{1}\right)$ is necessary for this transformation performance in the initial crystal. One this displacement could be performed by moving of a partial dislocation through a crystal resulting on a stacking fault (SF) ( $h h$-layers) in the initial crystal $3 \mathrm{C}$.

It is evident (fig. 1), that in the external case two different manners of transformation of one lattice into another are possible. In one case, each following displacement occurs next to the preceding one in two layers, i.e. coherently. It another case displacements occur all over a crystal independently on time and location of other displacements, i.e. noncoherently.

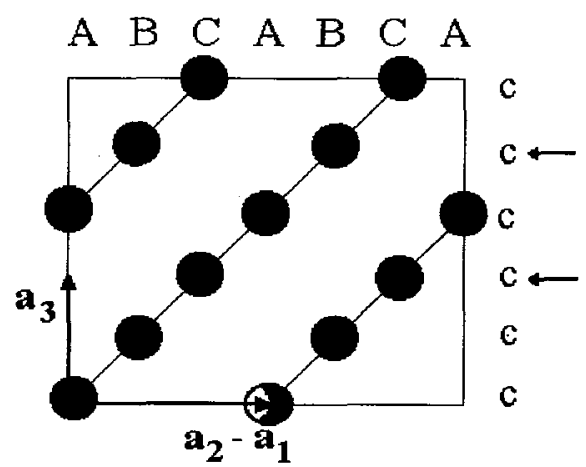

a)

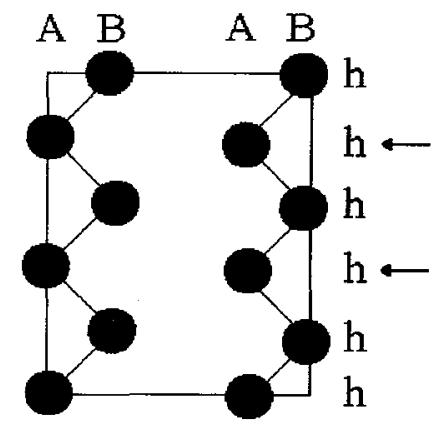

b)

Figure 1: Scheme of arrangement of close packed atomic layers in $3 \mathrm{C}$ (a) and $2 \mathrm{H} \mathrm{(b)-crystals.} \mathrm{Arrows} \mathrm{show}$ the places where displacement is necessary to be produced in order to transform one structure into another

It is clear, that in case of coherent appearance of displacements, disordering in packing of $2 \mathrm{H}$-structure atomic layers would be minimal. Long-range order (LRO) will be preserved in atomic layers arrangement in a crystal, as far as, only random disordering are possible due to non-coherent appearance of some displacements at the initial moment of transformation in one crystal, which appeared to be the centers of coherent appearance of displacements further. The disordering can occur as a result of joining of these areas. It can be presented on the microscopic level as origination of some nuclei of a new phase of a structure $2 \mathrm{H}$.

Under the non-coherent appearance of displacements probability of the disordering appearance in the atomic layers packing peculiar to a $2 \mathrm{H}$-crystal would be much higher. Besides, the probability of appearance of another statistics of space arrangement of faults is very important in this case due to crystallographic barriers, preventing the ordering of noncoherently appeared h-layers [5], resulting in formation of a short-range order (SRO) of atomic layer packing.

So, study of structural changes on two levels : microscopic and mesoscopic ones, is necessary for creation of a subsequent mechanism of close-packed polytypes formation under phase transformation of a displacive type. Study of the microscopic level should eliminate the way of realization of single displacements (if they are performed due to moving of partial dislocations, then what are the reasons). Study of the mesoscopic level should demonstrate the cause of displacements appearance on some distance from each other and the reason of sequence of displacements appearance. It is clear, that this distribution is artificial and assumes the mutual influence of the above levels. 
The results on mesoscopic level in particular are considered in this paper, i.e., study of the rules of space arrangement of displacements, resulting in polytype structure formations in alloys.

As far as, the investigation of polytype formation in situ is appeared to be difficult in some cases, we can utilize the advantage of difference between statistic laws of polytypes disordering as a result of performance of the coherent and non-coherent mechanisms of displacement appearance, which allows us to reconstruct with certain accuracy the transformation process.

Method of direct high-resolution of a lattice provides great possibility for solution of this problem. However, the experiments performed proved high efficiency of X-ray diffraction method for this purpose [6-7].

\section{X-RAY DIFFRACTION IN ODD STRUCTURE}

Let's assume that displacements in a crystal can be produced not only in a periodical way and a repulsion between the nearest neighbouring $c c$-layers on the distance four layers also exists. The probability to find the next $\mathrm{cc}$ on the distance $\mathrm{r}$ from a previous one can be presented in a way shown in fig.2a. This is simplest model of ODD structure with SRO in structural elements space arrangement.

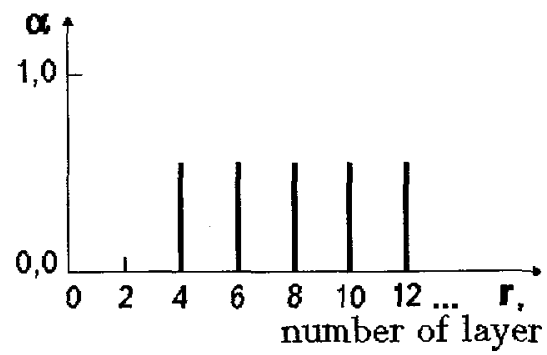

a)

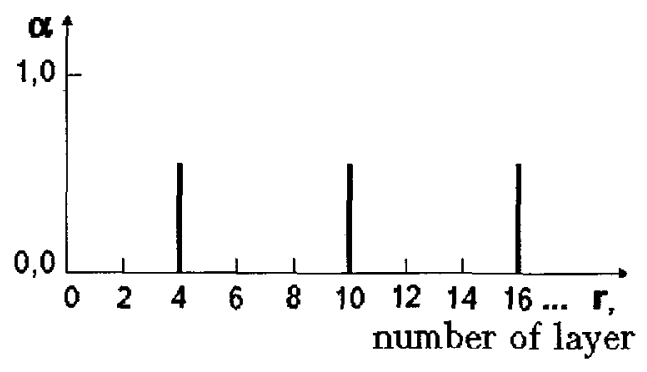

b)

Figure 2: The probability of a next $c c$-layer appearance in r layers from the nearest one in $h h h h .$. sequence in case of SRO (a). The same for case of LRO (b)

Changing in intensity distribution in a reciprocal lattice along the row with $\mathrm{H}-\mathrm{K} \neq$ $3 \mathrm{~N}$ where $\mathrm{N}=0, \pm 1, \pm 2, \pm 3 \ldots$, along which the diffuse scattering intensity is localized [9], caused by deviation in the atomic layers sequence is shown in fig. 3 for the different amounts of $\alpha$. One can see that intensity distribution between shifted and broadened peaks of $2 \mathrm{H}-$ structure becomes non-monotonous and additional peaks arise, when some characteristic values is reached. Their numbers and relative positions depend upon $r$. As the $\alpha$ increases halfwidth of peaks diminishes and they are shifted to sites of reciprocal lattice of a structure which is at $\alpha=1$. In example involved there are $18 \mathrm{R}(h h h h c c)$ - structure. Though diffraction pattern is characterized by existence of additional peaks typical for long periodical structure, even there is no distant order in arranging of $h h$ - and $c c$-layers

Let's consider a situation when $c c$-layers are arranged precisely in sites of a supperlattice i.e. at distances for example $1=4,10,16 \ldots$ from each other with probability $\alpha$ (fig. 2b). This is the simplest example of ODD structure with LRO in a structural elements space arrangement. 


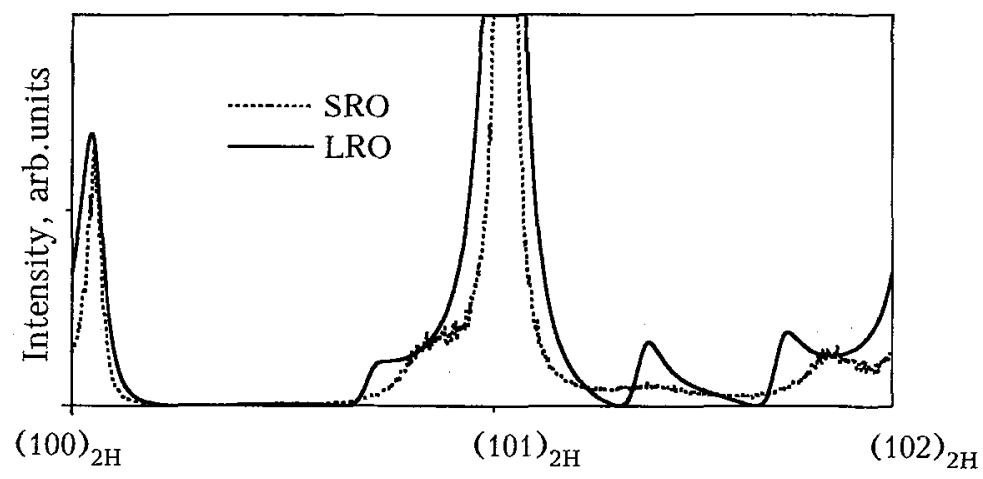

Figure 3: Intensity distribution along $10 \mathrm{~L}$ row in case of crystal with SRO and LRO of the same type (hhhhcc). Densities of $c c$-layer in both cases are equal 0.13 .

It was established, that on the contrary to SRO, the additional maxima appear at LRO at smaller values, and the maxima themselves are characterized by asymmetry. So, at the non-ideal LRO the diffraction pattern can be qualitatively similar to that one, which is peculiar to the ideal polytype.

Comparison of the intensity distribution in the reciprocal lattice for LRO and SRO exhibits that their intensity distributions are significantly different (fig. 3). Distances between diffuse scattering maxima demonstrate this difference. At LRO these distances are equal to some value or multiple of the one to it [11] when at SRO they are significantly different.

Thus spatial intensity distribution along the $H-K \neq 3 N$ reciprocal lattice directions allows to make a statistics of a spatial location of $h$ - and $c$-layers without a complete description of a crystal structure, which permits to gain knowledge about the displacement in the initial crystal, whether they are coherent or non-coherent.

\section{POLYTYPES FORMATION WITH A SRO IN ATOMIC LAYERS ARRANGEMENT}

While doping of some elements [12] in $\mathrm{Co}$, as $\mathrm{Ta}, \mathrm{Nb}, \mathrm{Sn}, \mathrm{Al}$, diffuse intensity of scattering by crystals of a low temperature polymorphous modification is continuously transformed (fig. 4).

The intensity distribution analysis in a reciprocal lattice proved that the alloys structure can be described by a probability scheme peculiar to SRO. In this case, the probability of appearance of $c$-layers in $h h .$. sequence linearly depend on the alloying element content (fig.5).

On the experimental data ground we came to the conclusion that the polymorphous transformation on the non-diffuse mechanism can be realized by a non-coherent displacements appeared in the initial structure.

It should be mentioned that SRO in the atomic layers arrangement of a lowtemperature polymorphous modification, e.g. alloy, is stable to the cooling rate and changes qualitatively in a good agreement with the alloying element concentration in a transformed solid solution. 


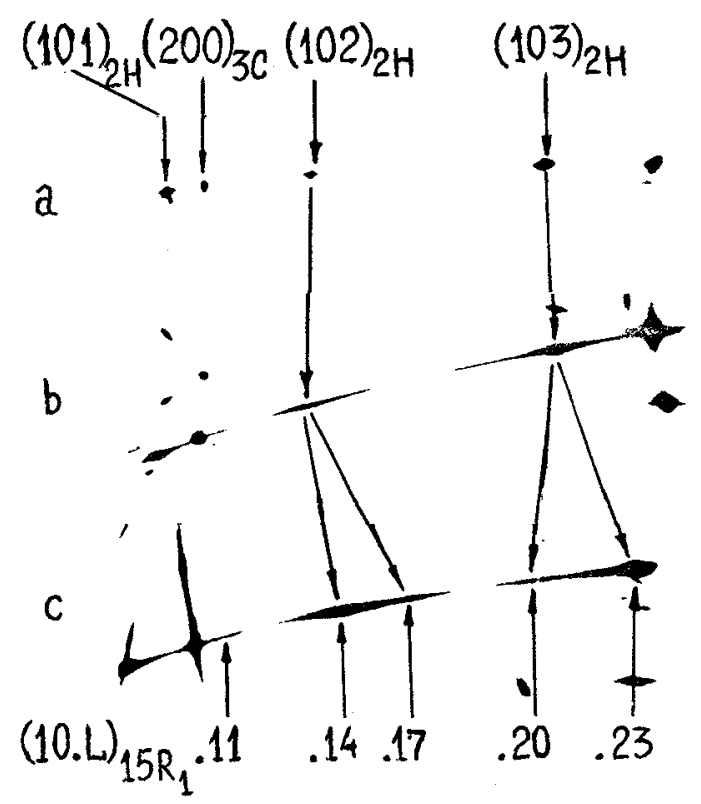

Figure 4: Fragments of $\mathrm{X}$-ray diffraction patterns demonstrate an intensity distribution along 10L row in case of a single crystal of alloys Co- 0.7 at.\% Ta (a); Co-1.7\% Ta (b); Co-2.8 \% Ta (c)

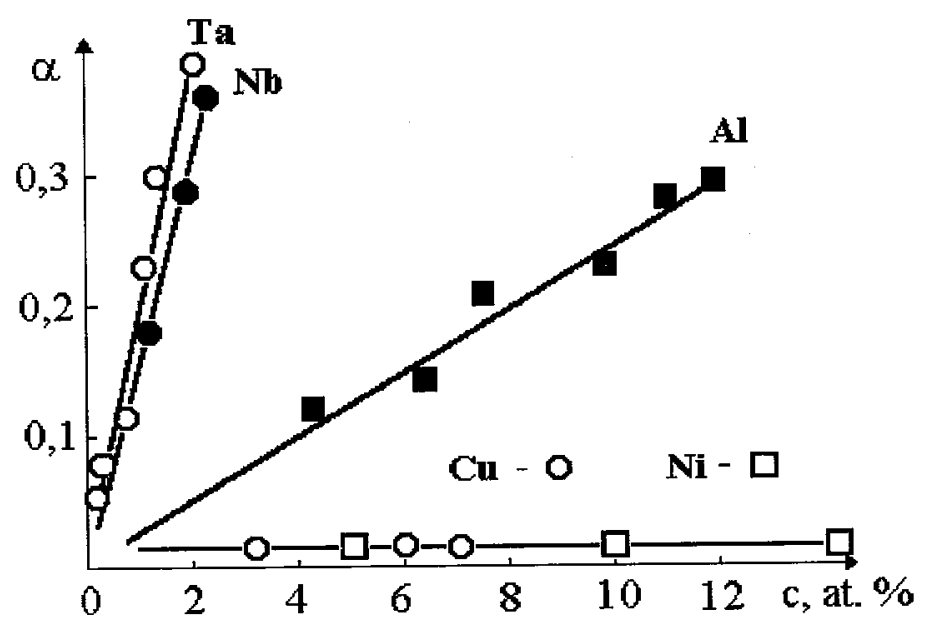

Figure 5: Relation between the probability of $c$-layer appearance in $h h . .$. sequence ( $h h h h c$-type SRO) and alloying element concentration in cobalt

\section{POLYTYPE STRUCTURE FORMATION WITH MOBILE IMPURITIES}

Under alloying of alloys with the elements with the high diffuse mobility under displacement transformation the qualitatively different case is observed. So, under doping of Co-alloys with carbon, which concentration is higher some critical one, the polytype 
structure changes qualitatively [13]. Diffuse scattering is characterized by a sharp appearance of a great number of additional maxima in the identity period of a reciprocal lattice with the carbon concentration change in an alloy beginning from some critical concentration (fig.6).

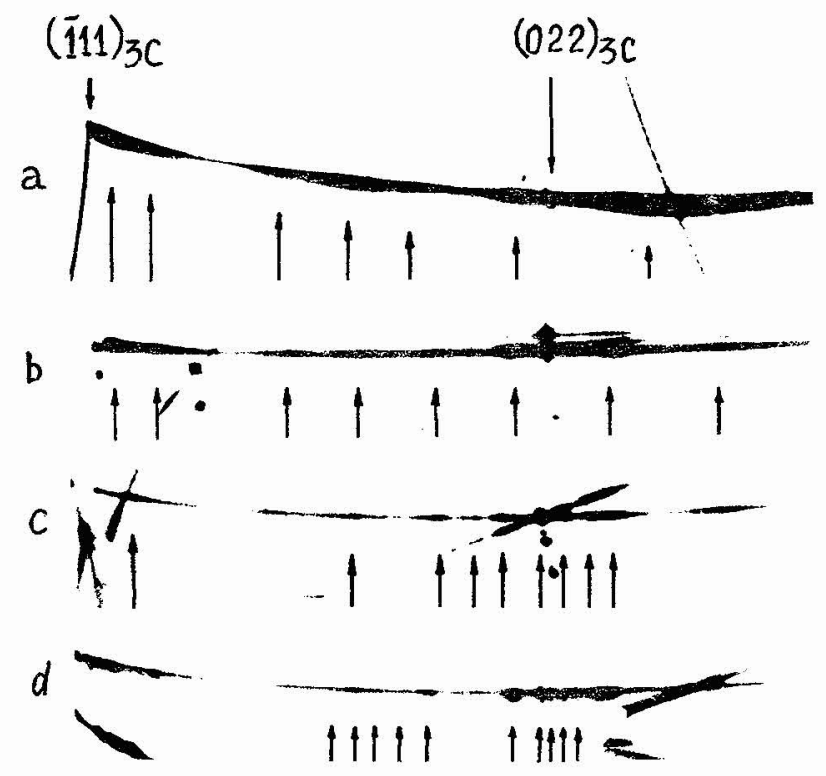

Figure 6: Fragments of $\mathrm{X}$-ray diffraction patterns demonstrate intensity distribution along 10L row in case of a single crystal of alloys Co-1.33 at.\%C (a), Co-1.69\%C (b); Co-2.08\% C (c); Co-2.17\% $\mathrm{C}$ (d) quenched in water from $1520 \mathrm{~K}$. Arrowed lines show the peaks of polymorphous modification lying along the row of $10 \mathrm{~L}-$ type reciprocal lattice, by which the correlation length and sequence of the atomic layer stacking was estimated

These polytypes can be described as structures with a correlation length of dozens in interplana distances. SRO of close packed atomic layers arrangement is changed at increasing of the carbon content in an alloy as:

$h h h h c \rightarrow h h h h c c c \rightarrow h h h h c c c c \rightarrow(h h h h c c c c)_{2} h h(c){ }_{10} h h c c c \rightarrow(h h h h c c c c)_{2}(c){ }_{3} h h(c)_{16} h h(c) 9 . \quad$ In their essence, these structural states can be presented as heterostructures, i.e. as a mixture of high-temperature and low-temperature parts pure Co modification or Co-alloy [14]. Absence of diffraction peculiarities of LRO exhibits the non-coherent displacement appearance also occurred at polytype formation.

First of all, the non-homogeneous displacements arrangement (of $h$-type layers) attracts the attention. Besides, these structures are appeared to be non-stable under their following cooling at lower temperature [15].

One of the possible reasons of these structures formation could be a direct interaction of impurities with LRO formed with displacements. Formation polytype mechanism on the mesolevel in this case could be presented as nucleation of a low temperature polymorphous modifications (of $h$-layers), which is accompanied with change of impurity concentration in the vicinity of them (e.g. due to different chemical potentials of atoms in $h$-and $c$-layers). As the result of the local increase of impurity concentration, free energy of $c$-layers could change so, that thermodynamic driving force, providing the $h$-layers growth due to new displacements will be significantly decreased. 
It is clear, that this will stimulate the appearance of new nuclei on some distance from the existing ones but not the ones appeared before, providing non-coherent displacement formation.

Experimental test of some aspects of this model is rather difficult. However, it is evidently that within this model the kinetics of polymorphous transformation is expected to effect on the polytype structure.

\section{POLYMORPHOUS TRANSFORMATION KINETICS EFFECT ON POLYTYPES FORMATION}

From the proposed polytypes formation mechanism follows that high mobility of an impurity polymorphous transformation is a very significant factor. In case of carbon these conditions could be gained at relatively low temperature. In case of impurity atoms the temperature range of transformation should be rather high.

Analysis of the state diagrams (fig. 7) showed, that solubility of an alloying element in $h$ layers (2H-structure) in $\mathrm{Co}-\mathrm{Si}$ and $\mathrm{Co}-\mathrm{Ge}$ systems is higher that in $c$-layers (3C-structure). It could result in redistribution of atoms of an alloying element under transformation and in local depleted neighboring to $h$-layers $c$-layers. As far as the polymorphous transformation temperature increases with the $\mathrm{Ge}$ and $\mathrm{Si}$ concentration (fig.7), the nearest neighboring $c$ layers are expected to be stabilized during concentrational fluctuation and new displacement will appear on some distance from the previous one. As the result, the configurations of ...hhchh... can originate, which transformation into $h h h h$ - sequence will be difficult under further cooling of an alloy.

As far as the living-time of concentrational fluctuation is limited, the rate of a crystal cooling should effect on the process above. Under slow cooling the concentrational inhomogeneities of an alloying elements considered above will disappear due to smoothing diffusion, stimulating $h h$-nucleus growth and thus, causing the $2 \mathrm{H}$ structure formation, peculiar to pure Co. Some transformation character could be expected also under fast cooling, when the concentrational inhomogeneities are not able to appear. Within some interval of cooling rate the correlatively arranged $c$-layers within $h h$-matrix can be expected.

The upper limit of the rate to a value, necessary for $c$-layer stabilization can be provided under depletion of a c-layer neighboring to the formed $h h$-layers by an impurity. In the first approximation this limit is equal to: $V \leq \beta c_{0} D \Delta U /\left(k T d^{2}\right)$, where $\beta=\partial T_{S} / \partial c ; T_{S}$ is a start temperature of polymorphous transformations ; $c_{0}$ is a element concentration in alloy; $\mathrm{D}$ is a diffusion coefficient, $\Delta U$ is an difference of energy in alloying $3 \mathrm{C}$ - and $2 \mathrm{H}$ structures; $\mathrm{k}$ is a Boltzman constant ; $T=T_{0}+\beta c_{0}, T_{o}$ is an equilibrium temperature of $3 \mathrm{C} \rightarrow 2 \mathrm{H}$ transformation; $\mathrm{d}$ is an interplanar distance. The unequality allows to evaluate the lowest limit value of the cooling rate under which single $c$-layers can be still stabilized and as a result, can form a heterocrystal.

Substitution of numerical values into the equation for Si and Ge allowed to evaluate the upper limit of cooling rates: $1.8 \cdot 10^{6} \mathrm{~K} / \mathrm{s}, 0,9 \cdot 10^{2} \mathrm{~K} / \mathrm{s}$ respectively.

Investigation of sample structures of $\mathrm{Co}-12.0$ at.\% $\mathrm{Ge}$ and $\mathrm{Co}-10$. at.\% Si alloys were performed within the interval of cooling rates $10^{-2} \ldots 10^{5} \mathrm{~K} / \mathrm{s}$.

The polytype structure depending on cooling rate of a sample is shown in fig. 8 .

A structure with SRO of a $h h c$-type is observed within the cooling rate interval of

$10^{-1} \ldots 10^{3}$. Under slower rates of cooling practically perfect $2 \mathrm{H}$-structure is formed and under rates higher $10^{4} \mathrm{~K} / \mathrm{sec}$ only a small amount of SF with a small correlation of mutual arrangement is in a structure. In $\mathrm{Co}-\mathrm{Si}$ alloy we did not manage to suppress the $c c c c \rightarrow h h c$ transformation accelerating the cooling rate to $10^{5} \mathrm{~K} / \mathrm{s}$. While slowing the cooling, the 


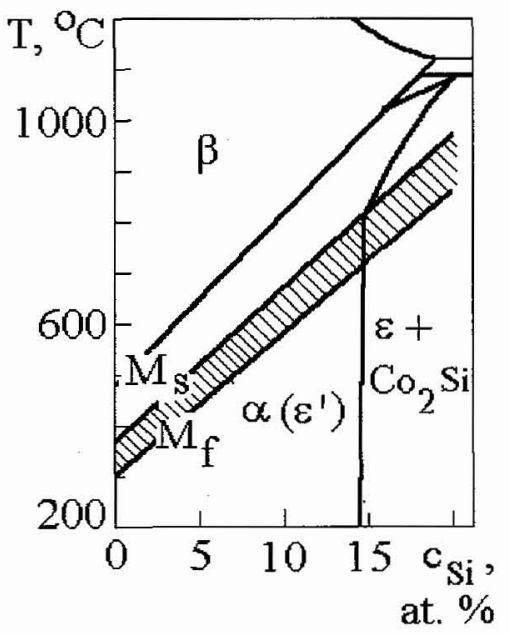

a)

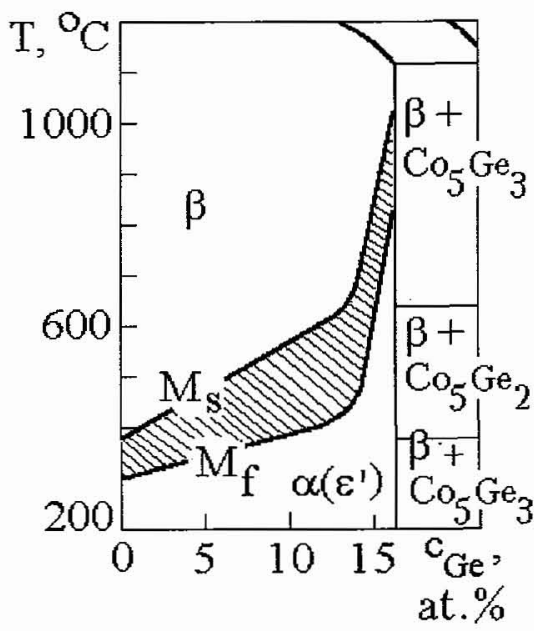

b)

Figure 7: Equilibrium state diagram for $\mathrm{Co}-\mathrm{Si}(\mathrm{a})$ and $\mathrm{Co}-\mathrm{Ge}(\mathrm{b})$ systems

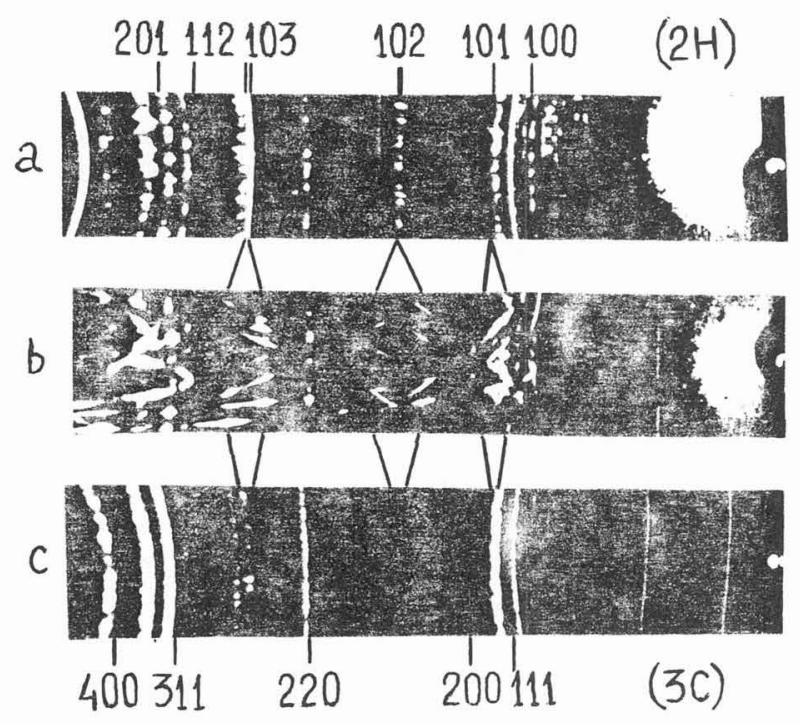

Figure 8: X-ray powder diffraction pattern for Co-Ge alloy cooled with rates $10^{-1} \mathrm{~K} / \mathrm{c}$ (a); $10^{3}$ (b); $10^{5}$ (c). Arrows show diffraction peaks of low temperature polimorphous modification structure

change of a transformation type and almost perfect $2 \mathbf{H}$-structure formation has been observed just at cooling rate of $10 \mathrm{~K} / \mathrm{s}$.

The experimentally obtained dependence of a probability of hhc configuration in a crystal on a cooling rate is shown in fig. 9 .

We have to take into consideration a threshold character of a kinetic dependence of a structural parameter, typical for nonequibibrium processes in self-organized systems. 


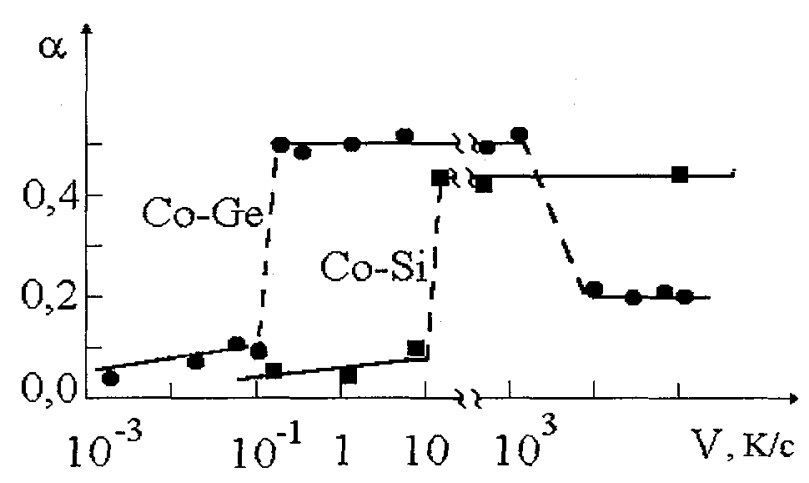

Figure 9: Probability of hhc-arrangement atomic layers appearance in a crystal of Co-10.0 at. \% Si and Co-at. \% $12.0 \mathrm{Ge}$ depending on cooling rate

\section{POLYTYPES FORMATION UNDER NON-EQUILIBRIUM CONDITIONS AS STACKING FAULTS SELF-ORGANIZING PROCESS}

Experimental results obtained under study of a cooling rate effect on a polytype structure permit to describe this process on the basis of kinetic equations for functions of stacking-faults distribution in impurity atoms.

Kinetic equations of a Fokker-Planck-type were proposed in paper [16], which are non-linear according to distribution functions and have solutions, which can correspond to non-equilibrium phase transformations into ordered states. Analyzing the formation conditions of non homogeneous distribution of stacking faults in solid solution we come to the conclusion that influence of an alloying element on SF ordering is as follows: first, due to interaction with the impurity atoms the redistribution of the latter occurs, and the potential of SF interaction with one another is ransomed and indirect interaction of SF via impurity atoms appears; second, if a solid solution is appeared to be unstable as to concentration waves formation, small content fluctuation can cause a SF distribution modulation.

For quantitative prediction of polytypes structure formed under non equilibrium conditions within the frames of a proposed approach, knowledge of potentials of SF interaction between each other is necessary as well as with impurity atoms and also interaction of impurity atoms with each other in real systems.

\section{CONCLUSION}

As the result of our investigation we came to the conclusion that under certain conditions (may be, alloying in case of Co, causing d-zone filling and weakening SF energy) alongside with a coherent mechanism of displacement formation, the qualitatively different modification character of polytype structure is in alloys with the mobile, under transformation, impurity atoms under non-equilibrium polymorphous transformation. 
Conditions of non-equilibrium polytypes formation under polymorphous transformations in alloy are expected to be: a) difference of chemical potentials of impurity atoms in $h$-and $c$-layers; b) rather high mobility of impurity atoms (comparing to the partial dislocation rate of a crystal, providing concentrational fluctuations in the vicinity of $h$-layers and their maintenance for some period of time (enough for new displacements formation in the nearest neighboring areas of crystals).

Some small deviations of parameters at limit values determining formation of a lowtemperature polymorphous modification may significantly effect on its structure, leading to a numerous polytype modifications, observed in alloys under, practically, same conditions.

\section{References}

[1] Nikolin B.I. Multilayer Structures and Polytypism in Metallic Alloys.( Izd.,Naukova Dumka, Kiev, 1994)

[2] Varlimont H., Dilay L., Martensitic Transformations in Copper-Silver- and Gold-Based Alloys(Pergamon Press, Oxford-New York-Toronto-Sydney, 1974)

[3] Otsuka K., Sakamoto H., Shimizu K., Acta Met. 27 (1979) 585-601.

[4] Martinov V.V., Tkachenko A.V., Khandros L.G., Metallofisika, 5 (1984) 59-63.

[5] Demin S.A., Nekrasov A.A., Ustinov A.I., Acta Metal. Mater., 41 (1993) 2091-2095.

[6] Pandey D., Lele S., Acta Metall 34 (1986) 405-413.

[7] Pandey D., Lele S., Acta Metall 34 (1986) 415-424.

[8] Olikhovskaya L.A., Rood' A.D., Ustinov A.I., Chuistov K.V., Metalofizika 10 (1988) 3237.

[9] Vilson A., X-Ray Optics (London, 1949).

[10] Ustinov A.I., Ukrainskii Fizichnyi Jurnal, 33 (1988) 253-261.

[11] Ruschits S.V., Mirzaev D.A., Kristallografiya, 24 (1979) 1142-1149.

[12] Skorodzievskii., Rood' A.D., Ustinov A.I., Chuistov K.V.,Scripta Met., 22 (1988) 307312.

[13] Skorodzievskii V.V., Ustinov A.I., Chuistov Izvestiya Akademii Nayk SSSR "Metally" 51986) 142-147.

[14] Demin S.A., Nekrasov A.A., Ustinov A.I.,Olikhovskaya L.A., Metallofizika 12 (1990) 69-75.

[15] Rood' A.D., Ustinov A.I., Chuistov K.V., Cryst.Res.Thechnol. 24 (1989) 395-403.

[16] Gaevskii A.Yu., Ustinov A.I., Metallofizika, 10 (1988) 103-109. 